\title{
POLARIZED LIGHT DETECTION IN SPIDERS
}

\author{
MARIE DACKE ${ }^{1, *}$, THUY A. DOAN ${ }^{2}$ AND DAVID C. O'CARROLL ${ }^{2}$ \\ ${ }^{1}$ Department of Zoology, University of Lund, Helgonavägen 3, S-223 54 Lund, Sweden and ${ }^{2}$ Department of Zoology, \\ University of Washington, Box 351800 Seattle, WA 98195, USA \\ *Author for correspondence (e-mail: marie.dacke@zool.lu.se)
}

Accepted 19 April 2001

\begin{abstract}
Summary
We describe here the detection of polarized light by the simple eyes of spiders. Using behavioural, morphological, electrophysiological and optical studies, we show that spiders have evolved two different mechanisms to resolve the e-vector of light. Wolf spiders (Lycosidae), are able to turn in response to rotation of a polarized pattern at the zenith of their visual fields, and we also describe a strip in the ventral retina of the principal (anterio-median) eyes that views this location and has receptors tiered into two

have evolved a pair of lensless secondary eyes for the detection of polarized light. These two eyes, each sensitive to orthogonal directions of polarization, are perfectly designed to integrate signals from the larger part of the sky and cooperate to analyse the polarization of light. Built-in polarizers help to improve signal purity. Similar organisation in the eyes of several other spider families suggests that these two mechanisms are not restricted to only a few families.
\end{abstract} layers. This provides each pair of receptors with a similar optical solution to that provided by the 'dorsal rim area' of the insect compound eye. In contrast, gnaphosid spiders
Key words: polarized light detection, simple eye, spider, vision, retinal tiering.

\section{Introduction}

Spiders live in a complex, three-dimensional world. Webbuilders, for example, construct lethal realms in which only they can survive. Others, such as the jumping spiders (Salticidae), are agile, arboreal hunters that roam horizontally or vertically in pursuit of prey and may pounce on it from a great distance (Jackson and Blest, 1982). Many other hunting spiders, such as the so-called 'wolf spiders' (Lycosidae), are in fact sit-and-wait predators (Ford, 1978). These spiders often build a permanent burrow or retreat and lurk nearby to await the approach of prey. Finding the way back to their retreat and orienting themselves within the web are complex tasks for which spiders employ many adaptations, from trailing silk lifelines to a variety of sensory systems.

Unlike the compound eyes of many other arthropods, spiders evolved simple eyes as their main visual organs. These are highly developed in some species, with acuity that rivals that of primates (Land, 1985). Not only do spiders have the bestdeveloped simple eyes of arthropods, but they also luxuriate in their multiplicity, having up to four pairs. Morphological and embryological differences allow these eyes to be arranged into two groups. A single pair of principal eyes, the anterio-median (AM) pair, is directed forwards. In some species, these eyes have a small field of view, which in part is compensated by a movable retina (Land, 1985). The remaining three pairs, the so-called secondary eyes, are named for their relative position on the head. The anterio-lateral (AL) eyes flank the principal eyes and are positioned in front of and below the posteriomedian (PM) and posterio-lateral (PL) eyes (Fig. 1). With few exceptions, the secondary eyes have a reflecting tapetum lining the back of the eye, while the AM eyes lack a tapetum in all species (Land, 1985).

With a multitude of eyes oriented in different directions, some spiders achieve an extended view of the world without having to move. In theory, having so many eyes would also make it possible to devote each pair to unique roles. In some species, the visual fields of one or more eye pairs are completely overlapped by those of others, suggesting that they are indeed specialized for different tasks (Land, 1985; Land and Barth, 1992). Some hunting spiders use at least one pair of secondary eyes for movement or target detection, and then rely on their principal (AM) eyes to identify the object (Schmid, 1998; Land, 1971). In others, the secondary eyes themselves may be enormous (particularly in nocturnal species), have high acuity and play a dominant role in prey discrimination (Land, 1985; Rovner, 1993), leaving the function of the overlapping AM eyes less obvious.

In this paper, we consider the possibility that the evolution of multiple eye pairs has allowed some spider eyes to become specialized for the task of orientation to patterns of polarized light in the sky. Together with new findings, we present an overview of polarized light detection and polarizing optics in the principal and secondary eyes of spiders. 


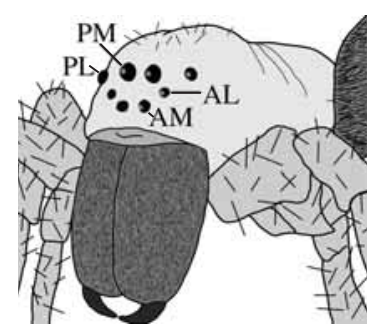

Fig. 1. The simple eyes of spiders are named after their relative position on the head. The comparative size and layout of the anteriomedian (AM), anterio-lateral (AL), posterio-median (PM) and posterio-lateral (PL) eyes does, however, vary with species.

\section{Optical basis for polarization sensitivity}

Although their retinal organization is very variable, all spider eyes possess microvillar photoreceptors similar to those of other arthropods (Blest, 1985). Such photoreceptors have the potential to be sensitive to the plane of polarization of light, because the light-absorbing pigments are organized so that the whole structure preferentially absorbs light vibrating parallel to the long axes of the microvilli (Snyder, 1973; Laughlin et al., 1975; Goldsmith and Wehner, 1977). In insects, the microvilli lie on one face of the receptive segment to form a single light-absorbing structure, the rhabdomere. In typical insect rhabdomeres, the microvilli are parallel along the entire length of the receptive segment, so that that the whole photoreceptor is sensitive to the e-vector of polarized light (Snyder, 1973; Laughlin et al., 1975).

Opponency between different receptors tuned to different angles of polarization facilitates the analysis of polarization, independent of the brightness of the stimulus (Nilsson and Warrant, 1999). In insects, specialized structures have evolved for this task, such as the well-studied 'dorsal rim area' within which photoreceptors are sensitive to short-wavelength blue or ultraviolet light and have rhabdomeres with orthogonal microvilli (Burghause, 1979; Labhart, 1980). Although large receptive fields are not essential for polarization vision, these polarization analysers typically have very poor spatial acuity (Aepli et al., 1985; Labhart, 1983). It has been argued that large receptive fields allow integration of signals over large regions of sky, permitting e-vector detection even when the blue sky is partially obscured by cloud or vegetation (Labhart et al., 1984; Labhart et al., 1992; Labhart et al., 2001; Meyer and Labhart, 1993).

Orthogonally arranged microvilli have also been described in the eyes of spiders (Blest and Carter, 1988; Blest and O'Carroll, 1990; Blest et al., 1981; Dacke et al., 1999; Eakin and Brandenburger, 1971; Land, 1969; Melamed and TrujilloCenóz, 1966; Schröer, 1971; Schröer, 1974). In addition, the reflecting sheet of crystals forming the tapetum of the secondary eyes can act as a polarizer to enhance the polarization sensitivity of the entire eye (Dacke et al., 1999).

Behavioural evidence for polarized light detection by spiders comes from three families, the ground-dwelling lycosid Arctosa variana (Magni et al., 1964), the agelenid funnel web spider Agelena labyrinthica (Görner, 1962; Görner and Claas, 1985) and the gnaphosid spider Drassodes cupreus (Dacke et al., 1999). Of these three, only the gnaphosid appears to use its secondary eyes for the task.

\section{Evidence for polarized light navigation by wolf spiders}

A series of experiments from the early 1960s suggested that some wolf spiders (Lycosidae) orient themselves using polarized light (Papi and Tongiorgi, 1963; Magni et al., 1964). Using behavioural analysis of the path taken by spiders placed in an arena, these experiments showed that spiders tended to move in a direction that would lead them back to their permanent retreat. They further showed that, when the pattern of polarized light was altered experimentally, the spiders altered their preferred direction accordingly. By selectively ablating the eyes, further experiments showed that the AM eyes were primarily responsible for this behaviour (Magni et al., 1964).

Despite a detailed anatomical and physiological investigation, however, subsequent work failed to find either a structural basis for this polarization sensitivity in the AM eyes (Bacetti and Bedini, 1964; Melamed and Trujillo-Cenóz, 1966) or any convincing physiological evidence for these eyes being strongly polarization sensitive (Magni et al., 1965). Enigmatically, the same studies were able to find a consistent microvillar orientation and demonstrate some polarization sensitivity in the secondary eyes, even though the latter were not, apparently, involved in the behaviour.

\section{Wolf spiders exhibit an optomotor response to rotation of polarized light}

To further investigate the possibility that lycosids orient themselves in relation to the e-vector of polarized light, we designed an experiment based on qualitative observations made in the earlier studies. Spiders (Pardosa tristis) were waxed to a stick by the cephalothorax and placed with their legs in contact with a lightweight ball (circumference $120 \mathrm{~mm}$ ) that was free to rotate on an air cushion (Fig. 2A). An ultraviolet-rich (Xenon) light source was viewed by spiders through a $72 \mathrm{~mm}$ diameter linear polarizer (Hoya, $50 \%$ cut-off at $375 \mathrm{~nm}$ ) that could be rotated by a DC motor and placed at the zenith (Fig. 2A). To minimize polarized reflections from objects within the spider's fields of view, the spider was suspended at the centre of a vertically oriented cylinder, $22.5 \mathrm{~cm}$ in diameter and $15.5 \mathrm{~cm}$ high, lined with fibrous white architectural paper. The orientation of the cylinder ensured that polarized grazing-incidence reflections from the paper were directed to below the spider's position. Motion of the spider on the ball was analysed by a 2-dimensional optical motion sensor (a Microsoft optical mouse connected to a Macintosh computer). This was positioned so that we could distinguish between 'progress' (motion in a direction that would maintain the spider's orientation relative to the polarization stimulus), and 'rotation' (yaw). Spiders were stimulated to run on the ball by the touch of a probe on the abdomen or rear legs. Typical 


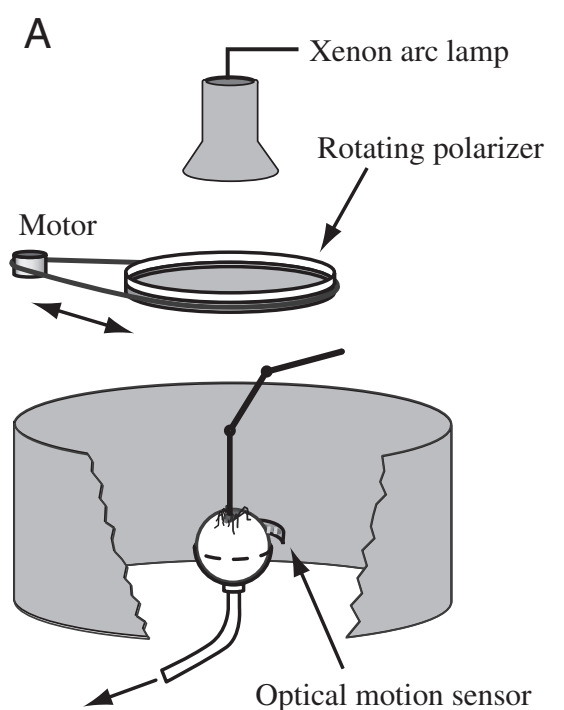

To compressed air
B

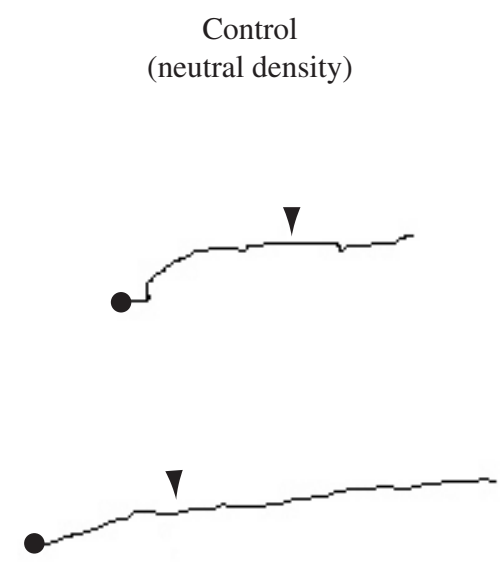

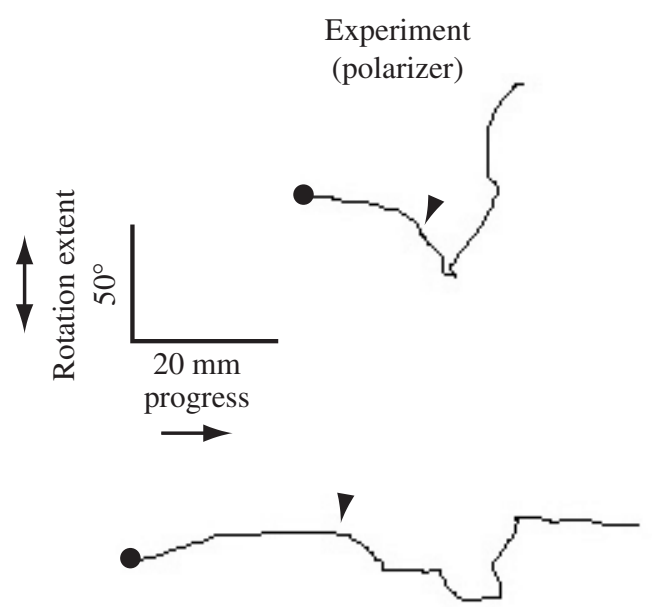

Fig. 2. (A) Diagram of the apparatus used to demonstrate that a dorsal region of the field of view is used for polarization analysis by lycosids. (B) Raw responses of spiders (Pardosa tristis) to rotation of a polarizer (right) and a neutral density filter (left). The filled circles indicate the starting point of the spider. The arrowheads indicate the commencement of filter rotation. For further details, see text.

running bouts lasted between 5 and $10 \mathrm{~s}$ and rotation of the filter was only turned on after spiders had been in motion for 2 or more seconds. Data were only collected while the spider continued to run on the ball.

Typical responses of the spider to rotation of the polarizer are shown in Fig. 2B. Progress of the spider is indicated by the track from left to right, while down and up in these figures represent clockwise or anticlockwise rotation, respectively, by the spider. Following the commencement of continuous rotation of the polarizer at $50^{\circ} \mathrm{s}^{-1}$ (indicated by the arrowheads in Fig. 2B), spiders responded rapidly (typically within a few hundred milliseconds) by increasing their rotation of the ball. This stands in stark contrast to the case when we replace the polarizer with a control stimulus (an unpolarized neutral density filter, with similar mean transmission) (Fig. 2B).

To quantify this difference in degree of compensatory rotation, we selected two small (30 pixels wide) 'progress' windows, corresponding to approximately $30^{\circ}$, or $10 \mathrm{~mm}$ of forward travel (if the spider was running freely on a flat surface). These prerotation and perirotation analysis windows were symmetrically located either side of a zone 20 pixels wide at the point of commencement of rotation (arrowheads in Fig. 2B). This allowed us to account for uncertainty in the exact rotation commencement point (which was marked onto each trace by hand). For any point on the spider's response path, the slope of a tangent to the path defines the strength of the turning response (yaw) relative to forward progress. A slope of 0 would represent a straight trajectory, whereas large values indicate that the spider is turning 'on the spot', either clockwise or anti-clockwise. We measured the response by calculating this local rotational 'slope', defined as the height (in pixels) of the path at each of the 30 successive pixels traced out by the spider's progress within each window. Our initial analysis suggested that the spider's turning direction was random (clockwise versus anti-clockwise) so we took the mean absolute value of the 30 local slopes as a measure of the response.

Fig. 3 shows that rotation of the polarizer elicited a highly significant (Student's paired $t$-test) increase in the degree of rotation, while rotation of the neutral density filter evoked no significant response. Since the most obvious cue available to

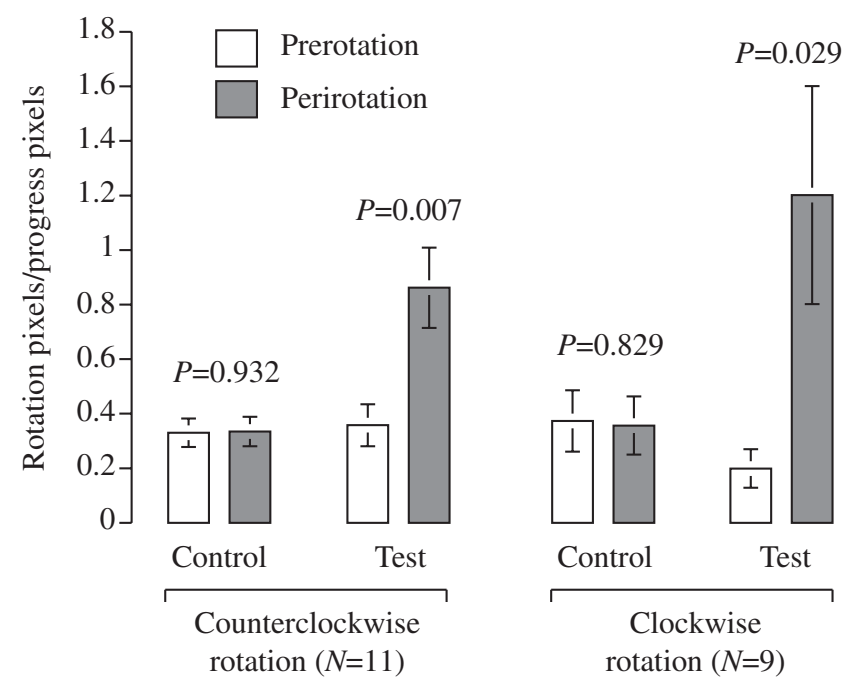

Fig. 3. Averaged responses of wolf spiders to the stimulus described in Fig. 2. Responses before (open columns) and after (grey columns) rotation of either a neutral density filter (control) or a polarizer (test) are shown for either clockwise $(N=9)$ or counterclockwise $(N=11)$ rotation of the filter. In both cases, $P$ values show the statistical significance of the difference in rotational response of the spider (Student's paired $t$-test). Values are means \pm S.E.M. 
distinguish the polarizer from the control is the e-vector of light, our results suggest that wolf spiders are able to perceive this signal and use it to generate an optomotor response. Our stimulus was placed symmetrically at the dorsal pole in relation to the body axis, subtending an angle of approximately $30^{\circ}$. This region of space is viewed only by the AM eyes and the most dorsal margins of fields of view of the PM and PL eyes (Land, 1985). Although we have not, as yet, used selective ablation of the eyes to demonstrate that this behaviour is indeed mediated by the AM eyes, the earlier studies strongly suggest that this is the case (Magni et al., 1964). From these results we can expect the analyser of polarized light to be located in the most ventral part of the retina in the AM eyes, since simple eyes form an upside-down image on the retina. Do the AM eyes of hunting spiders have an organization consistent with this prediction?

\section{Retinal tiering in the principal eyes of hunting spiders}

Earlier studies suggest that the typical AM retina in lycosids and other hunting spiders has a uniform monolayer of photoreceptors, each with a polygonal shape and an irregular number (typically 4-6) of rhabdomeres on the outer faces of each receptive segment (Bacetti and Bedini, 1964; Blest, 1985). Adjacent rhabdomeres are not separated by glial cells, so the entire retina has the appearance of a 'network' of rhabdomeres, with no consistent microvillar orientation (Blest, 1985). This organization is poorly suited to polarization analysis.

Exceptions to this rule, however, are the well-studied AM eyes of jumping spiders (Salticidae). In this family, specialization of different eye pairs is taken to an extreme. The AM eyes have small, densely packed photoreceptors and a long focal length (Land, 1969; Land, 1985). The extremely high acuity that results from this combination is augmented by a tiered structure, with four layers of photoreceptors each sensitive to different wavelengths of light (Land, 1969; Blest et al., 1981; Blest et al., 1990), suggesting a role for these eyes in colour discrimination (Land, 1985).

This remarkable tiered structure was believed to have evolved originally as a solution to the problem of chromatic aberration resulting from the optics of long focal length (Blest et al., 1990; Land, 1969) rather than for polarization analysis. Nevertheless, the most distal layer is ultraviolet-sensitive (Blest et al., 1981) and microvilli of this layer are oriented horizontally in the peripheral retina and vertically in the central retina (Eakin and Brandenburger, 1971; Blest and Carter, 1988). As mentioned above, this orthogonal organization is consistent with a potential role in polarization analysis.

Are such retinal specializations unique to salticids? To address this question, we have now re-examined the structure of the AM retina in a number of hunting spider species, including lycosids. We find that several families, including the Lycosidae, Pisauridae, Oxyopidae and Thomisidae (subfamily Misumeninae), indeed have local regions in which the AM retina is tiered into two layers (Blest and O'Carroll, 1990). In misumenine thomisids, the tiered region is small, with just a dozen or so photoreceptors. The tiered regions in lycosids and their sister family the Pisauridae are, however, much larger and have a structure highly suggestive of a role in polarization analysis.

\section{The lycosid AM retina contains a ventral strip of orthogonal, tiered photoreceptors}

The tiered region in lycosids is a small, strip-like sub-region in the ventral retina, while the remainder of the retina has a 'networked' organization similar to the typical spider arrangement. In the tiered region, however, photoreceptors have square profiles and only two parallel rhabdomeres, on opposite faces of the cell (Fig. 4). To avoid confusion, we will

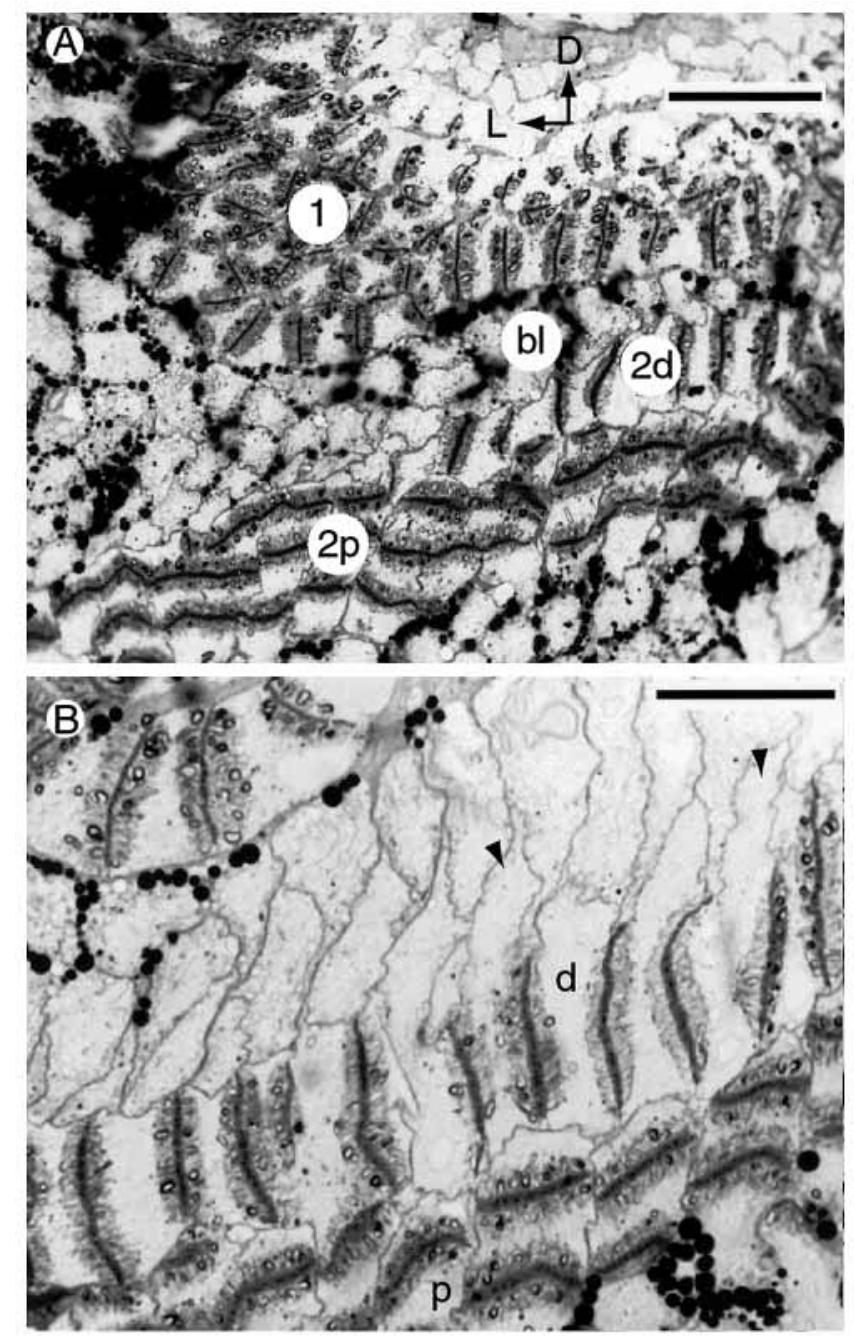

Fig. 4. Light micrograph showing transverse sections through the ventral anterio-median retina of a lycosid wolf spider (species A). (A) Low-power view, showing type 1 receptors (1) and both distal (2d) and proximal (2p) type 2 receptors from the tiered region. The blind strip (bl) between these layers in clearly visible in this section. Scale bar, $55 \mu \mathrm{m}$. D, dorsal; L, lateral. (B) A higher power view of the tiered region, showing orthogonal type 2 receptors in the distal (d) and proximal (p) layers and longitudinal, vertically extended intermediate segments of the distal receptors (arrowheads). Scale bar, $25 \mu \mathrm{m}$. 
Table 1. Mean dimensions and inter-receptor angles $(\Delta \phi)$ for the three photoreceptor types (type 1 and both proximal and distal layers of tiered type 2 receptors) in the principal eyes of four lycosid species

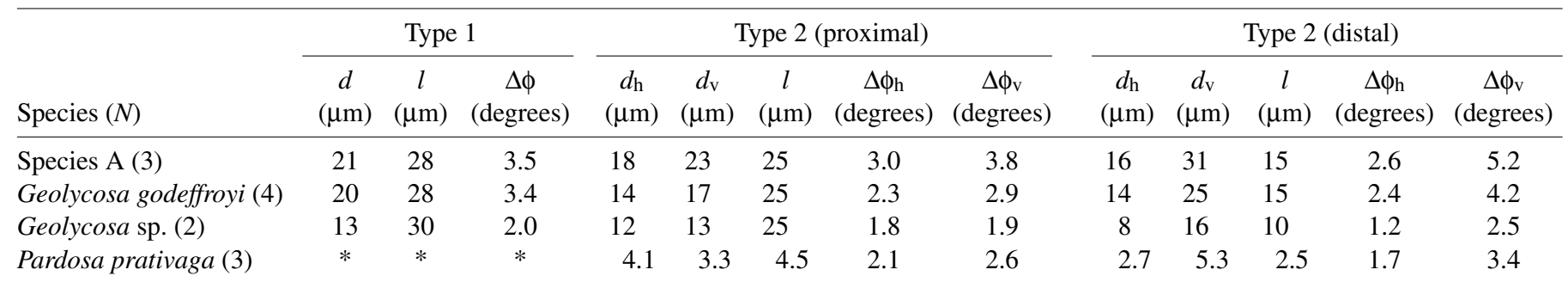

* No data obtained for this region.

$d$, rhabdom diameter; $l$, rhabdom length; $\Delta \phi$, inter-receptor angle; $d_{\mathrm{h}}$, horizontal (medio-lateral) rhabdom diameter; $d_{\mathrm{v}}$, vertical (dorsoventral) rhabdom diameter; $\Delta \phi_{\mathrm{h}}$, horizontal (medio-lateral) inter-receptor angle; $\Delta \phi_{\mathrm{v}}$, vertical (dorso-ventral) inter-receptor angle.

refer to the more common, networked type receptive segments with multiple rhabdomeres as 'type 1' and those from this ventral region, with paired rhabdomeres, as 'type 2' (see Table 1).

Type 2 photoreceptors are tiered into two layers. The tiered strip is laterally extended and slightly crescent-shaped, tilted slightly so that the medial edge extends further dorsally than the lateral edge (Fig. 4). In transverse sections through this part of the retina, photoreceptors of the distal layer have vertically oriented rhabdomeres with extended, rectangular cross sections (Fig. 4). The intermediate segments (the part of the cell between the receptive segment and the axon in the optic nerve) project dorsally from the receptive segments (arrowheads in Fig. 4B). All other photoreceptors in the eye (or in the AM eyes of other spiders) project posteriorly. Consequently, longitudinal sections through the tiered strip show no continuation of the intermediate segments of the distal layer below the proximal layer (Fig. 5). Furthermore, just dorsal to the tiered region, the intermediate segments of the distal layer bend at right angles, in line with the optic axis, and then exit the retina, suggesting that the distal layer is formed from photoreceptors that were originally more dorsal (close to the optic axis). This organization is summarized in Fig. 5C.

The organization described above has two direct consequences. Firstly, where intermediate segments of the distal layer converge to exit the retina, they form a small 'blind' strip devoid of receptive segments. This is analogous to the optic disc of vertebrate retinae. The 'blind' region clearly divides the tiered region from the type 1 receptors (Fig. 4, Fig. 5). Second, in bending over to lie above the proximal layer, the distal photoreceptors have rotated through $90^{\circ}$. The two layers therefore not only receive input from identical points in space but have orthogonally arranged microvilli. With the potential to code the e-vector of light independent of absolute luminance or local contrast, the two layers of type 2 receptors would thus make an ideal polarization analyser.
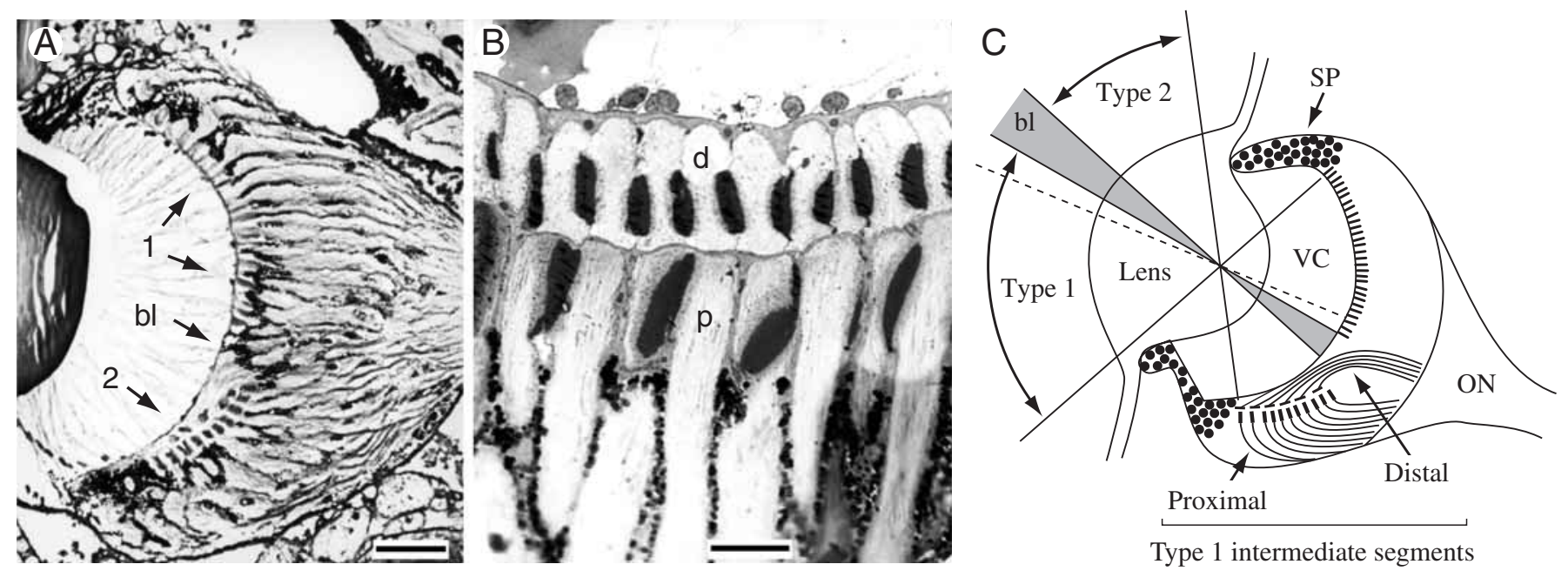

Fig. 5. Longitudinal sections through the anterio-lateral retinae of a lycosid (species A). (A) A low-power view of a vertical section showing the positions of type 1 (1) and type 2 (2) receptors and the blind region (bl). Dorsal is up, and ventral down in this figure. Scale bar, $35 \mu \mathrm{m}$. (B) A higher power view of a horizontal section through the ventral tiered region. The distal (d) and proximal (p) layers are clearly visible, with no continuation of the intermediate segments of the distal layer through the proximal layer. Scale bar, $15 \mu \mathrm{m}$. (C) Summary of the organization of the retina as seen in longitudinal section, showing the fields of view of each region. The shaded region shows an approximate projection through the nodal point of the lens of the blind region (bl) that divides the fields of view of the type 1 and type 2 receptor regions. $\mathrm{VC}$, vitreous cells; SP, screening pigment; ON, optic nerve. 
We found this retinal organization in five lycosid species, Geolycosa godeffroyi, an undescribed Geolycosa species and a further unidentified lycosid species (termed here species A), all collected in South Australia. Incomplete series of sections in fewer orientations were obtained from the British species Pardosa prativaga and Alopecosa pulverulenta. However, all sections obtained are consistent with the organization described above. In particular, both layers of type 1 photoreceptors are clearly visible in the ventral region of the retina, so that this organization seems to be common to all lycosids.

\section{Large fields of view and poor spatial vision in the tiered part of the retina}

The inter-receptor angle, $\Delta \phi$, is an anatomical measure of the acuity of an eye (Land, 1985). This can be calculated from the receptor spacing determined histologically, and the focal length measured optically using the hanging drop technique (Homann, 1928; Land, 1985). The results of such measurements are given in Table 1. Because of the rectangular transverse profiles of distal layer type 2 receptive segments, the inter-receptor angle measured parallel to the long axes of rhabdomeres (the 'vertical' inter-receptor angle, $\Delta \phi_{\mathrm{v}}$ ) is typically larger than that measured perpendicular to this orientation (the 'horizontal' inter-receptor angle, $\Delta \phi_{\mathrm{h}}$ ). Indeed, their diameter along the dorso-ventral axis $\left(d_{\mathrm{v}}\right)$ is greater than their depth (receptor length), again suggesting that these photoreceptors are 'turned on their side'. The inter-receptor angles are large compared with the 1-1.5 degrees estimated for lycosid secondary eyes or in typical insects (Land, 1985), suggesting that the AM eyes have comparatively poor acuity, especially in the tiered strip, where $\Delta \phi$ is close to $5^{\circ}$. The very low $F$-numbers (focal length/lens aperture) measured in the same four species (species A, 0.70; G. godeffroyi, 0.73; Geolycosa sp., 0.71; P. prativaga, 0.75) confirm that these eyes are designed for efficient light-gathering, rather than for high acuity (Land, 1985; Warrant and McIntyre, 1991).

In addition to poor anatomical resolution, the photoreceptors lack any optical isolation from their neighbours by screening pigment, and the rhabdomeres of adjacent photoreceptors are also contiguous. In a low- $F$-number eye such as these, most light focused onto the retina enters through the margins of the lens and therefore enters photoreceptors at a large angle. In the absence of screening pigment, this light will rapidly stray into the adjacent photoreceptors, with the net effect of further increasing the receptive field size (acceptance angle) of each (Warrant and McIntyre, 1991). A further optical 'blurring' effect must result from the fact that the three photoreceptor types (type 1, distal and proximal type 2) lie at three discrete depths relative to the centre of curvature of the cornea (Fig. 5). Low $F$-numbers give a small 'depth of field', and all three layers could thus not possibly lie in the plane of focus.

The short focal lengths and semi-spherical retinae found in all four lycosid species suggest that the overall fields of view of the principal eyes are large, covering an extensive region of the space towards which they are directed (forwards and upwards in most species) and overlapping at the front. This is in accordance with the fields of view of different pairs of eyes in the lycosid Arctosa variana (Magni et al., 1964). Because the tiered region occupies the ventral margin of the retina, however, it is directed skywards, towards the most dorsal part of this field (Fig. 5). It is in this part of the visual world that we find the cues for polarized light navigation (Wehner, 1989).

\section{Polarized light detection in secondary eyes}

While specialized structures such as we describe from the principal eyes have been found in only a few families of hunting spiders, the secondary eyes of many other species are ideally suited for the detection of polarized light. They generally have photoreceptors with microvilli oriented in a consistent direction, and in some species, acute spatial vision seems deliberately to have been abolished by having the retina in front of the focal plane of the lens (Land, 1985). In addition, spiders of many families have polarizing tapeta that may further facilitate the detection of light polarization (see below). Despite this wealth of indications, only a single species, the gnaphosid Drassodes cupreus, has been demonstrated to use its secondary eyes for polarized light navigation (Dacke et al., 1999).

\section{Polarized light navigation in Drassodes cupreus involves a unique compass organ}

As the sun sets, the gnaphosid spider Drassodes cupreus leaves its well hidden and tightly spun silk nest to search for prey. The spider then returns to its shelter, provided it is given a polarization pattern similar to that of the evening sky. As soon as this pattern is removed, or the secondary eyes are covered with black, opaque paint, the spider can no longer find its way home (Dacke et al., 1999). This leaves Drassodes cupreus as the single known navigator shown to use its secondary eyes for this task.

The morphology and electrophysiology of the secondary eyes of this spider reveal a series of specializations that work together to provide an ideal compass organ. The majority of the photoreceptors in the PM eyes of Drassodes cupreus have their microvilli arranged parallel to the long axis of the eye (Fig. 6A,B) so that the whole eye will be maximally sensitive to a single direction of polarization. This characteristic retinal arrangement is found in all the secondary eyes of the spider. As to be expected for receptors with such well-aligned microvilli, intracellular recordings reveal high polarization sensitivity ratios (Snyder, 1973), in this case as high as 9.1 (Fig. 7) (Dacke et al., 1999).

Together, the three pairs of secondary eyes in Drassodes cupreus have a field of view that covers the entire sky above the horizon (Fig. 8) and the eyes have the potential to be used for polarized skylight navigation. Since they are oriented at different angles, each eye will respond to different directions of polarization. As mentioned above, reliable analysis of the e-vector of polarized light requires a comparison of signals from cells with different polarization axes, but with the same 
field of view. While the AL and PL eyes are sensitive to different polarization axes, the overlap in their visual fields is not extensive. However, the fields of view of the PM eyes are entirely overlapping and are also centred on the zenith, where the highest degree of polarization can be found around dusk (Dacke et al., 1999).

In addition, the PM eyes have lenses that are little more than flat, transparent windows, providing no significant refracting power (Dacke et al., 1999). This would provide the photoreceptors with a poorly focused image and, therefore, a large receptive field. Combined with their overlapping fields of view and nearly orthogonal preferred e-vector directions for the left and right eyes, the pair of PM eyes is thus an ideal analyser of the simple dusk polarization pattern. At this time of the day, the sky is polarized mainly in one direction, and no direct sunlight interferes with a wide-field analyser. The AL and PL eyes might very well still serve another polarization analysing task so far unknown to us.

\section{Many spider secondary eyes are equipped with polarizing optics}
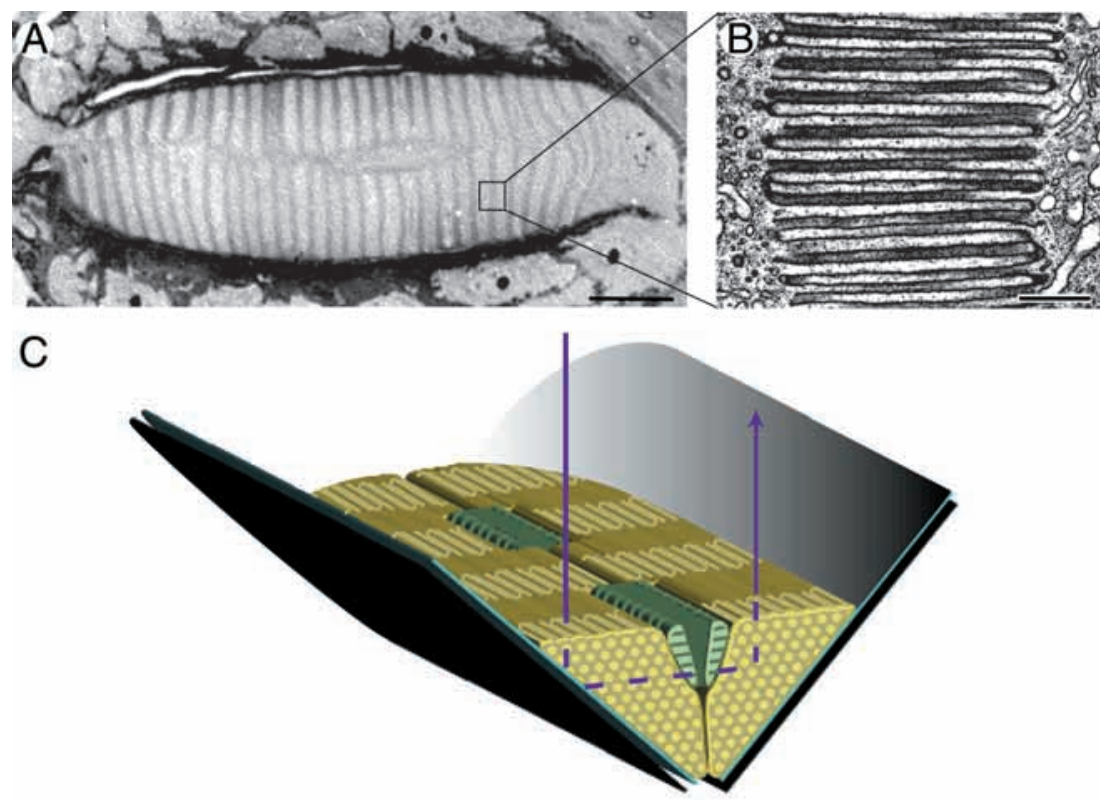

Fig. 6. Structure of the posterio-median eye of Drassodes cupreus. (A) Tangential section (light micrograph; scale bar, $1 \mu \mathrm{m}$ ) through the retina of the PM eye revealing a regular rhabdomeral arrangement. (B) Electron micrograph of the boxed region in A, showing the parallel microvillar arrangement found over the bigger part of the retina. Scale bar, $500 \mathrm{~nm}$. (C) Drawing of the 'canoe-shaped tapetum' and the retina, with one possible path of light through the eye (arrow). The front end of the eye is cut off to expose interior structures. (Modified from Dacke et al., 1999.)

Weak lenses are not a novelty restricted to the secondary eyes of Drassodes cupreus; many spiders have lenses that form images well below the retina (Land, 1985). Such strange optics are still an enigma. In these eyes, as in the eyes of Drassodes cupreus, the tapetum is typically shaped a little like a boat, hence the name 'canoe-shaped tapetum' (Fig. 6C). This form of tapetum is extremely common, being found in 21 other families of spider (Land, 1985). Given our finding that the PM eyes of Drassodes cupreus are polarization analysers, is it possible that this type of eye is adapted to a similar role in other spider families? As many of the spiders with this type of eye are web-builders, it has been suggested that they could make use of a compass for the orientation of their webs (Marshall, 1999).

This possibility is further suggested by our recent finding that the polarization sensitivity of these eyes in Drassodes cupreus is not entirely accounted for by the structure of the photoreceptors themselves: the eyes also contain polarizing optics (Dacke et al., 1999). Light reflected from the tapetum

Fig. 7. Intracellular recordings in the retina of a posterio-median eye of the spider Drassodes cupreus. Left and right response-intensity curves are recorded with light polarized parallel (Para) and perpendicular (Perp) to the long axis of the eye, respectively. The log intensity shift of 0.96 between the two curves translates to a polarization sensitivity of 9.1 (a unitless quantity). (This figure was published as supplementary information in Dacke et al., 1999; http://www.nature.com/nature/journal/v401/n6752/suppinfo/401470a 0.html). of the PM eyes becomes polarized, with five times more light reflected parallel to the long axis of eye than perpendicular to it. As this direction of polarization coincides with the direction of maximal sensitivity of the receptors, it boosts the polarization signal by effectively attenuating the signals to which the photoreceptors are least sensitive. The only structures in the eye that could act as polarizing filters are the photoreceptors themselves and the tapetum. However, since the absorption of light by the retina would tend to polarize light along the short axis of the eye, orthogonal to the axis of polarization observed, we conclude that the tapetum itself acts

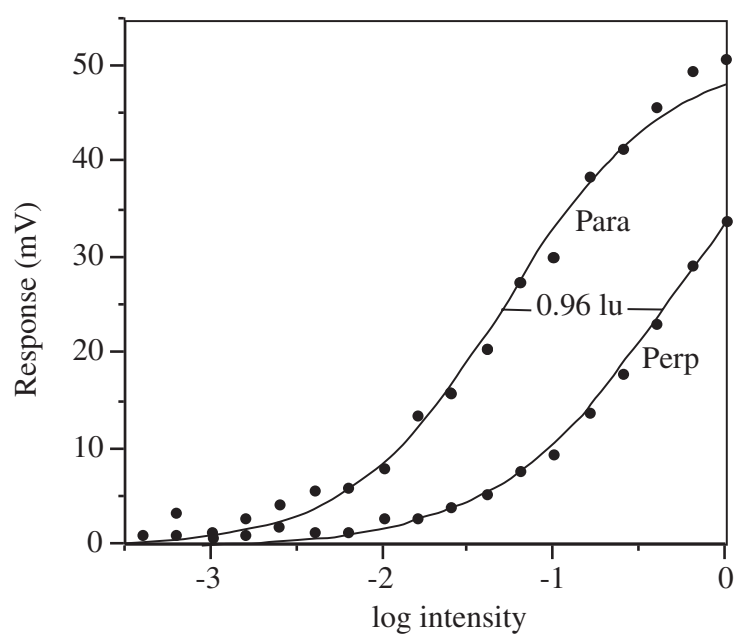




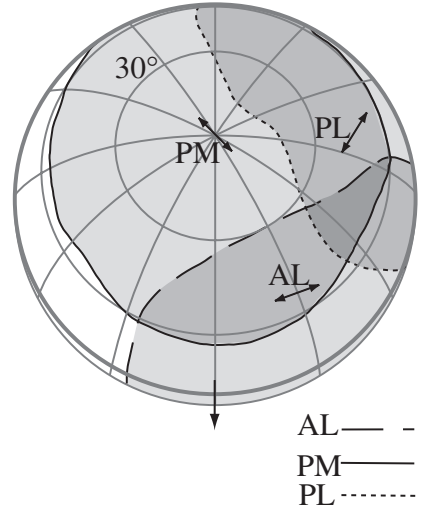

Fig. 8. Fields of view of the left set of secondary eyes of Drassodes cupreus. The fields are plotted onto a globe with the spider at the centre. The pole of the grid is straight up, and the one-ended arrow marks the longitudinal axis of the spider. The two-ended arrows indicate the direction of polarization to which each of the three eyes is most sensitive. Note the large and almost circular field of view of the posterio-median eye. AL, anterio-lateral; PL, posterio-lateral; PM, posterio-median.

as a polarizing reflector. The exact mechanism by which this selective reflection occurs is not yet known.

Might it be possible that the tapetum of other spiders also enhances polarized light detection? To answer this question, we measured the degree of polarization of the light reflected back from the secondary eyes of a number of families possessing canoe-shaped tapeta. We then examined the morphology of the PM eyes of at least one member of each family. The polarization measurements and the histology were according to the method described by Dacke et al. (Dacke et al., 1999). For comparison, identical measurements were also made on hunting spiders (Lycosidae, Thomisidae) with a grateshaped type of tapetum. These families have previously been shown to have well-focused eyes (Land, 1985).

The data obtained (Table 2) show that, in most of the eyes with a canoe-shaped tapetum, the reflections from one or more pairs of eyes are polarized. However, of the families studied, only the Gnaphosidae capitalise on this feature, by having retinae consisting almost entirely of photoreceptors with microvillar orientation parallel the plane of polarization reflected from the eyes. It is also in this family that we find the highest degree of polarization upon reflection. In the other families, the microvilli are arranged in several directions over the retina. Local arrangements could still support the analysis of polarized light in these other species of spider, but no obvious part of the retina has yet been identified for such a purpose. It is possible that variability in the degree of polarization of reflections is due to variability in the orientation of the guanine crystals that form the tapetum. Further work, using electron microscopic techniques, is required to establish whether this is the case.

Polarizing optics are not restricted to canoe-shaped tapeta (Table 2). The grid-shaped tapeta of crab spiders (Thomisidae, Misumeninae) also have the ability to polarize light upon
Table 2. The polarization of light measured in the reflection from secondary eyes in nine families of spiders with canoeshaped or grid-shaped tapeta

\begin{tabular}{llccc}
\hline Family & \multicolumn{1}{c}{ Species } & PM & PL & AL \\
\hline Gnaphosidae & Zelotes latreillei & 1.0 & 3.7 & 1.9 \\
& Drassodes cupreus & 5.3 & 4.1 & 5.3 \\
& Haplodrassus signifer & 2.5 & 2.6 & 2.8 \\
Theridiidae & Steatoda bipunctata & 2.9 & 1.7 & 2.6 \\
& Acareanae lunata & 2.8 & 2.5 & 2.5 \\
Aranenidae & Araneus diadematicus & 1.0 & 1.9 & 2.6 \\
& Zygiella X-notata & 3.1 & 2.1 & 2.3 \\
& Cersidia prominens & 1.2 & 1.2 & 2.1 \\
Agelenidae & Agelena labyrinthica & 1.2 & 3.0 & 2.1 \\
& Tegenaria campestris & 1.3 & 2.2 & 4.6 \\
Clubionidae & Clubionia lutescens & 2.4 & 2.1 & 2.3 \\
Segestridae & Segestria senoculata & 4.2 & 1.5 & 2.7 \\
Linyphiidae & Linyphia trangularis & $*$ & 2.8 & 2.5 \\
Thomisidae $\neq$ & Xysticus cristatus & 3.1 & 2.1 & 2.3 \\
Lycosidae $\neq$ & Pirata hygrophilus & 1.1 & 1.2 & 1.3 \\
& Throcosa terricola & 1.1 & 1.3 & 1.2
\end{tabular}

The values given are mean ratios $(N=3)$ between the intensity of light in the direction of polarization and in the direction $90^{\circ}$ to it. Hence, a value of 1.0 denotes a totally unpolarized reflection.

* No data obtained for this eye.

†rid-shaped tapetum.

$\mathrm{PM}$, posterio-median eye; PL, posterio-lateral eye; AL, anteriolateral eye.

reflection. However, in these eyes, we found the majority of the receptors to be arranged perpendicular to the direction of polarization, a design that reduces both the total sensitivity and the polarization sensitivity. Receptors with microvilli arranged at an angle up to $90^{\circ}$ to this general direction were also found in the periphery of these eyes. Together, these receptors could possibly be used for polarization analysis. The lycosids also have a grid-shaped tapetum (Bacetti and Bedini, 1964), but light polarization is nearly absent (Table 2).

To conclude, it seems that, while the poorly focused secondary eyes of many spiders possess polarizing optics, with the notable exception of the gnaphosid Drassodes cupreus the overall structure in many cases suggests that they are not optimized for the analysis of polarized light. The roles of many of these secondary eyes remain enigmatic, with their polarizing optics only adding to the mystery.

\section{Concluding remarks}

The luxury of having eight eyes has allowed lycosid and gnaphosid spiders to devote eyes to the detection of polarized light. The photoreceptors in the specialized structures of these eyes are directed skywards, receive an underfocused image and have poor spatial acuity. Polarized light analysers in spiders thus show striking parallels to the dorsal rim area found in the compound eyes of many insects. However, having a completely different eye design, spiders face a problem unfamiliar to insects. In the insect dorsal rim area, 
each ommatidium contains rhabdomeres with orthogonal microvillar orientations, such that the ratio of the responses of the two cell classes will code the e-vector of incoming light at each optical sampling point irrespective of the absolute luminance or local contrast (Burghause, 1979; Labhart, 1980). In simple eyes, each photoreceptor normally occupies a unique axial location in space, so that this type of analysis is not possible. The ratio of the outputs of two adjacent cells with orthogonal microvilli and different fields of view would depend on local contrasts as well as the e-vector of incoming light. In lycosid and gnaphosid spiders, this problem has been solved by two different, but equally effective, mechanisms.

In lycosids, orthogonal receptors are tiered in two layers. This provides each axial pair of cells with a similar optical solution to that provided by compound optics, since the two analysers are now co-axial. Not only does this provide receptors in the two layers with a co-axial view of the sky, but the distal layer could potentially improve the polarization sensitivity of the proximal layer through its selective absorption of light orthogonal to its preferred direction (Snyder, 1973). The only problem with such a system is that, since the polarization sensitivity of distal receptors would not be 'perfect', they would also screen the receptors in the proximal layer, reducing their absolute sensitivity. Such screening effects could, in turn, be minimized by having very short receptive segments, which is exactly what we observe in the distal layer (Table 1). In simple eyes with very low $F$ number optics, such as these, the absolute sensitivity of even short photoreceptors would still be very high (Land, 1985).

The gnaphosid spiders have come up with a slightly more drastic answer to the problem. By reducing the lens power of their polarization-sensitive eyes to a transparent window, individual photoreceptors will have almost as large a visual field as that of the whole eye. Coupled with an eye position that gives the two eyes completely overlapping visual fields, a reliable polarization analysis could then be obtained by opponent input summed across the left and right eye, which are oriented at $90^{\circ}$ to one another. Such integration across the entire eye is possible because of the parallel arrangement of microvilli across the whole retina. However, a limitation to such a solution is that it will work only at dusk and dawn, when one single direction of polarization is present across the sky. Integration over the greater part of the sky during the day, when the polarization pattern is more complex, would only weaken the signal. Hence, for polarized light navigation during the day, the lycosid solution is superior.

Despite the remarkably diverse solutions to the detection of polarized light by the simple eyes of spiders, their use in orientation is known from only three families. While the morphological requirement for polarized light analysis exists in many more families, it is not known whether these spiders actually use their eyes for this purpose. With their unique webs, many spiders create an animated world full of other cues for orientation, and vision may play only a subordinate role that is hard to isolate.
We thank Dan Nilsson and Eric Warrant for critically reading the manuscript and Rita Wallén for expert technical assistance. We thank Armin Hinterwirth for his wonderful diagrams and his help with experiments on lycosids. Financial support came from the Swedish Natural Science Research Council (M.D.) and the Mary Gates Foundation for Undergraduate Research (D.O'C., T.D.).

\section{References}

Aepli, F., Labhart, T. and Meyer, E. P. (1985). Structural specializations of the cornea and retina at the dorsal rim of the compound eye in hymenopteran insects. Cell Tissue Res. 239, 19-24.

Baccetti, B. and Bedini, C. (1964). Research on the structure and physiology of the eyes of a lycosid spider. I. Microscopic and ultramicroscopic structure. Arch. ital. Biol. 102, 97-122.

Blest, A. D. (1985). The fine structure of spider photoreceptors in relation to function. In Neurobiology of Arachnids (ed. F. G. Barth), pp. 79-102. Berlin, Heidelberg, New York: Springer Verlag.

Blest, A. D. and Carter, M. (1988). Post-embryonic development of the principal retina of a jumping spider. II. The acquisition and reorganization of rhabdomeres and growth of the glial matrix. Phil. Trans. R. Soc. Lond. B 320, 505-515.

Blest, A. D. and O'Carroll, D. (1990). The evolution of the tiered principal retinae of jumping spiders (Araneae: Salticidae). In Neurobiology of Sensory Systems (ed. R. Naresh Singh and N. J. Strausfeld), pp. 155-170. New York: Plenum Press.

Blest, A. D., Hardie, R. C., McIntyre, P. and Williams, D. S. (1981). The spectral sensitivities of identified receptors and the function of retinal tiering in the principal eyes of a jumping spider. J. Comp. Physiol. 145, 227-239.

Blest, A. D., O'Carroll, D. and Carter, M. (1990). Comparative ultrastructure of Layer I receptor mosaics in principal eyes of jumping spiders: the evolution of regular arrays of light guides. Cell Tissue Res. 262, 445-460.

Burghause, F. (1979). Die strukturelle Spezialisierung des dorsalen Augenteils der Grillen (Orthoptera, Grylloidea). Zool. Jb. Physiol. 83, 502-525.

Dacke, M., Nilsson, D.-E., Warrant, E. J., Blest, A. D., Land, M. F. and O'Carroll, D. C. (1999). Built-in polarizers form part of a compass organ in spiders. Nature 401, 470-473.

Eakin, R. M. and Brandenburger, J. L. (1971). Fine structure of the eyes of jumping spiders. J. Ultrastruct. Res. 37, 618-663.

Ford, M. J. (1978). Locomotory activity and the predation strategy of the wolf spider Pardosa amentata (Clerk) (Lycosidae). Anim. Behav. 26, 31-35.

Goldsmith, T. H. and Wehner, R. (1977). Restrictions on rotational and translational diffusion of pigment in the membranes of a rhabdomeric photoreceptor. J. Gen. Physiol. 70, 453-490.

Görner, P. (1962). Die Orientierung der Trichterspinne nach polarisiertem Licht. Z. Vergl. Physiol. 45, 307-314.

Görner, P. and Claas, B. (1985). Homing behaviour and orientation in the funnel-web spider, Agelena labyrinthica Clerck. In Neurobiology of Arachnids (ed. F. G. Barth), pp. 275-297. Berlin, Heidelberg, New York: Springer Verlag.

Homann, H. (1928). Beitrage zur Physiologie der Spinnenaugen. I. Untersuchungsmethoden. II. Das Sehvermogen der Salticiden. Z. Vergl. Physiol. 7, 201-269.

Jackson, R. R. and Blest, A. D. (1982). The distances at which a primitive jumping spider makes visual discriminations. J. Exp. Biol. 97, 441-445.

Labhart, T. (1980). Specialized photoreceptors at the dorsal rim of the honeybee's compound eye: polarizational and angular sensitivity. J. Comp. Physiol. 141, 19-30.

Labhart, T. (1983). The electrophysiology of visual cells in different eye regions of the ant Cataglyphis. Experientia 39, 637.

Labhart, T., Hodel, B. and Valenzuela, I. (1984). The physiology of the cricket's compound eye with particular reference to the anatomically specialized dorsal rim area. J. Comp. Physiol. A 155, 289-296.

Labhart, T., Meyer, E. P. and Schenker, L. (1992). Specialized ommatidia for polarization vision in the compound eye of cockchafers, Melolontha melolontha (Coleoptera, Scarabaeidae). Cell Tissue Res. 268, 419-429.

Labhart, T., Petzold, J. and Helbling, H. (2001). Spatial integration in polarization-sensitive interneurones of crickets: a survey of evidence, mechanisms and benefits. J. Exp. Biol. 204, 2423-2430.

Land, M. F. (1969). Structure of the retinae of the principal eyes of jumping 


\section{M. Dacke, T. A. Doan and D. C. O’CARroll}

spiders (Salticidae: Dendryphantinae) in relation to visual optics. J. Exp. Biol. 51, 443-470.

Land, M. F. (1971). Orientation of jumping spiders in the absence of visual feedback. J. Exp. Biol. 54, 119-140.

Land, M. F. (1985). The morphology and optics of spider eyes. In Neurobiology of Arachnids (ed. F. G. Barth), pp. 53-78. Berlin, Heidelberg, New York: Springer Verlag.

Land, M. F. and Barth, F. G. (1992). The quality of vision in the ctenid spider Cupiennius salei. J. Exp. Biol. 164, 227-242.

Laughlin, S. B., Menzel, R. and Snyder, A. W. (1975). Membranes, dichroism and receptor sensitivity. In Photoreceptor Optics (ed. A. W. Snyder and R. Menzel), pp. 237-259. New York: Springer Verlag.

Magni, F., Papi, F., Savely, H. E. and Tongiorgi, P. (1964). Research on the structure and physiology of the eyes of a lycosid spider. II. The role of different pairs of eyes an astronomicla orientation. Arch. Ital. Biol. 102, $123-136$.

Magni, F., Papi, F., Savely, H. E. and Tongiorgi, P. (1965). Research on the structure and physiology of the eyes of a lycosid spider. III. Electroretinographic responses to polarized light. Arch. Ital. Biol. 103, $146-158$

Marshall, J. (1999). Visual function: How spiders find the right rock to crawl under. Curr. Biol. 9, R918-R921.

Melamed, J. and Trujillo-Cenóz, O. (1966). The fine structure of the visual system of Lycosa (Araneae: Lycosidae). Part I. Retina and optic nerve. $Z$. Zellforsch. 74, 12-31.
Meyer, E. P. and Labhart, T. (1993). Pore canals in the cornea of a functionally specialized area of the honey bee's compound eye. Cell Tissue Res. 216, 491-501.

Nilsson, D.-E. and Warrant, E. J. (1999). Visual discrimination: seeing the third quality of light. Curr. Biol. 9, R535-R537.

Papi, F. and Tongiorgi, P. (1963). Innate and learned components in the astronomical orientation of wolf spiders. Ergeb. Biol. 26, 259-280.

Rovner, J. S. (1993). Visually mediated responses in the Lycosid spider Rabidosa rabida: The roles of different pairs of eyes. Mem. Queensland Mus. 33, 635-638.

Schmid, A. (1998). Different functions of different eye types in the spider Cupiennius salei. J. Exp. Biol. 201, 221-225.

Schröer, W.-D. (1971). Polarisationsempfindlichkeit rhabdomerialer Systeme in den Hauptaugen der Trichterspinne Agelena gracilens (Arachnida: Araneae: Agelenidae). Ent. Germ. 3, 88-92.

Schröer, W.-D. (1974). Zum Mechanismus der Analyse polarisierten Lichtes bei Agelena gracilens C. L. Koch (Araneae, Agelenidae). I. Die Morphologie der Retina der vorderen Mitteaugen (Hauptaugen). Z. Morph. Tiere. 79, 215-231.

Snyder, A. W. (1973). Polarization sensitivity of individual retinula cells. $J$. Comp. Physiol. 83, 331-360.

Warrant, E. J. and McIntyre, P. D. (1991). Strategies for retinal design in arthropod eyes of low F-number. J. Comp. Physiol. A 168, 499-512.

Wehner, R. (1989). The hymenopteran skylight compass: matched filtering and parallel coding. J. Exp. Biol. 146, 63-85. 